\title{
On the $N$-Solitons Solutions in the Novikov-Veselov Equation
}

Jen-Hsu CHANG

Department of Computer Science and Information Engineering, National Defense University, Tauyuan, Taiwan

E-mail: jhchang@ndu.edu.tw

Received October 01, 2012, in final form January 12, 2013; Published online January 20, 2013

http://dx.doi.org/10.3842/SIGMA.2013.006

\begin{abstract}
We construct the $N$-solitons solution in the Novikov-Veselov equation from the extended Moutard transformation and the Pfaffian structure. Also, the corresponding wave functions are obtained explicitly. As a result, the property characterizing the $N$-solitons wave function is proved using the Pfaffian expansion. This property corresponding to the discrete scattering data for $N$-solitons solution is obtained in [arXiv:0912.2155] from the $\bar{\partial}$-dressing method.
\end{abstract}

Key words: Novikov-Veselov equation; $N$-solitons solutions; Pfaffian expansion; wave functions

2010 Mathematics Subject Classification: 35C08; 35A22

\section{Introduction}

The Novikov-Veselov equation $[3,9,34,41]$ is defined by

$$
\begin{aligned}
& U_{t}=\partial_{z}^{3} U+\bar{\partial}_{z} U+3 \partial_{z}(V U)+3 \bar{\partial}_{z}\left(V^{*} U\right) \\
& \bar{\partial}_{z} V=\partial_{z} U, \quad \partial_{z} V^{*}=\bar{\partial}_{z} U
\end{aligned}
$$

When $z=\bar{z}=x$, we get the famous $\mathrm{KdV}$ equation $(U=\bar{U}=V=\bar{V})$

$$
U_{t}=2 U_{x x x}+12 U U_{x}
$$

The equation (1) can be represented as the form of Manakov's triad [24]

$$
H_{t}=[A, H]+B H
$$

where $H$ is the two-dimension Schrödinger operator

$$
H=\partial_{z} \bar{\partial}_{z}+U
$$

and

$$
A=\partial_{z}^{3}+V \partial_{z}+\bar{\partial}_{z}^{3}+\bar{V} \bar{\partial}_{z}, \quad B=V_{z}+\bar{V}_{\bar{z}}
$$

It is equivalent to the linear representation

$$
H \psi=0, \quad \partial_{t} \psi=A \psi .
$$

We see that the Novikov-Veselov equation (1) preserves a class of the purely potential selfadjoint operators $H$. Here the pure potential means $H$ has no external electric and magnetic fields. The periodic inverse spectral problem for the two-dimensional Schrödinger operator $H$ 
was investigated in terms of the Riemann surfaces with some group of involutions and the corresponding Prym $\Theta$-functions [5, 10, 22, 27, 28, 33, 37]. On the other hand, it is known that the Novikov-Veselov hierarchy is a special reduction of the two-component BKP hierarchy $[23,36,40]$ (and references therein). In [23], the authors showed that the Drinfeld-Sokolov hierarchy of D-type is a reduction of the two-component BKP hierarchy using two different types of pseudo-differential operators, which is different from Shiota's point of view [37]. Also, in [26], it is shown that the Tzitzeica equation is a stationary symmetry of the Novikov-Veselov equation. Finally, it is worthwhile to notice that the Novikov-Veselov equation (1) is a special reduction of the Davey-Stewartson equation [20, 21].

Let $H \psi=H \omega=0$. Then via the Moutard transformation [1, 29, 30, 31]

$$
\begin{aligned}
& U(z, \bar{z}) \longrightarrow \hat{U}(z, \bar{z})=U(z, \bar{z})+2 \partial \bar{\partial} \ln \omega \\
& \psi \longrightarrow \theta=\frac{1}{\omega} \int(\psi \partial \omega-\omega \partial \psi) d z-(\psi \bar{\partial} \omega-\omega \bar{\partial} \psi) d \bar{z}
\end{aligned}
$$

one can construct a new Schrödinger operator $\hat{H}=\partial_{z} \bar{\partial}_{z}+\hat{U}$ and $\hat{H} \theta=0$. We remark that the Moutard transformation (3) is utilized to construct the $N$-solitons solutions of the Tzitzeica equation [15].

The extended Moutard transformation was established such that $\hat{U}(t, z, \bar{z})$ and $\hat{V}(t, z, \bar{z})$ defined by $[13,25]$

$$
\hat{U}(t, z, \bar{z})=U(t, z, \bar{z})+2 \partial \bar{\partial} \ln W(\psi, \omega), \quad \hat{V}(t, z, \bar{z})=V(t, z, \bar{z})+2 \partial \partial \ln W(\psi, \omega),
$$

where the skew product (alternating bilinear form) $W$ is defined by

$$
\begin{aligned}
W(\psi, \omega)= & \int(\psi \partial \omega-\omega \partial \psi) d z-(\psi \bar{\partial} \omega-\omega \bar{\partial} \psi) d \bar{z}+\left[\psi \partial^{3} \omega-\omega \partial^{3} \psi+\omega \bar{\partial}^{3}-\psi \bar{\partial}^{3} \omega\right. \\
& +2\left(\partial^{2} \psi \partial \omega-\partial \psi \partial^{2} \omega\right)-2\left(\bar{\partial}^{2} \psi \bar{\partial} \omega-\bar{\partial} \psi \bar{\partial}^{2} \omega\right)+3 V(\psi \partial \omega-\omega \partial \psi) \\
& -3 \bar{V}(\psi \bar{\partial} \omega-\omega \bar{\partial} \psi)] d t
\end{aligned}
$$

will also satisfy the Novikov-Veselov equation.

In $[2,6,7,8]$, the rational solutions and line solitons of the Novikov-Veselov equation (1) are constructed by the $\bar{\partial}$-dressing method. To get these kinds of solutions, the scattering datum have to be delta-type and the reality of $U$ also puts some extra constraints on them. In [39], the singular rational solutions are obtained using the extended Moutard transformation (4); however, the non-singular rational solutions are constructed in [4].

Next, we construct Pfaffian-type solutions. Given any $N$ wave functions $\psi_{1}, \psi_{2}, \psi_{3}, \ldots, \psi_{N}$ (or their linear combinations) of (2) for fixed potential $U(z, \bar{z}, t)$, the $N$-step extended Moutard transformation can be obtained in the Pfaffian [1,31] (also see [12, 35])

$$
\begin{aligned}
& P\left(\psi_{1}, \psi_{2}, \psi_{3}, \ldots, \psi_{N}\right)=\left\{\begin{array}{l}
\operatorname{Pf}\left(\psi_{1}, \psi_{2}, \psi_{3}, \ldots, \psi_{N}\right) \text { if } N \text { even, } \\
\widetilde{\operatorname{Pf}}\left(\psi_{1}, \psi_{2}, \psi_{3}, \ldots, \psi_{N}\right) \text { if } N \text { odd, }
\end{array}\right. \\
& \operatorname{Pf}\left(\psi_{1}, \psi_{2}, \psi_{3}, \ldots, \psi_{N}\right)=\sum_{\sigma} \epsilon(\sigma) W_{\sigma_{1} \sigma_{2}} W_{\sigma_{3} \sigma_{4}} \cdots W_{\sigma_{N-1} \sigma_{N}}, \\
& \widetilde{\operatorname{Pf}}\left(\psi_{1}, \psi_{2}, \psi_{3}, \ldots, \psi_{N}\right)=\sum_{\sigma} \epsilon(\sigma) W_{\sigma_{1} \sigma_{2}} W_{\sigma_{3} \sigma_{4}} \cdots W_{\sigma_{N-2} \sigma_{N-1}} \psi_{\sigma_{N}},
\end{aligned}
$$

where $W_{\sigma_{i} \sigma_{j}}=W\left(\psi_{\sigma(i)}, \psi_{\sigma(j)}\right)$ is defined by the skew product (4). The summations $\sigma$ in (5) and (6) run from over the permutations of $\{1,2,3, \ldots, N\}$ such that $\sigma_{1}<\sigma_{2}, \sigma_{3}<\sigma_{4}, \sigma_{5}<\sigma_{6}$, $\ldots$ and $\sigma_{1}<\sigma_{3}<\sigma_{5}<\sigma_{7}<\cdots$, with $\epsilon(\sigma)=1$ for the even permutations and $\epsilon(\sigma)=-1$ for the odd permutations. Then the solution $U$ and $V$ can be expressed as [1]

$$
U=U_{0}+2 \partial \bar{\partial}\left[\ln P\left(\psi_{1}, \psi_{2}, \psi_{3}, \ldots, \psi_{N}\right)\right], \quad V=V_{0}+2 \partial \partial\left[\ln P\left(\psi_{1}, \psi_{2}, \psi_{3}, \ldots, \psi_{N}\right)\right],
$$


and the corresponding wave function is

$$
\varphi=\frac{P\left(\psi_{1}, \psi_{2}, \psi_{3}, \ldots, \psi_{N}, \vartheta\right)}{P\left(\psi_{1}, \psi_{2}, \psi_{3}, \ldots, \psi_{N}\right)}
$$

where $\vartheta$ is an arbitrary wave function different from $\psi_{1}, \psi_{2}, \psi_{3}, \ldots, \psi_{N}$.

The paper is organized as follows. In Section 2, we obtain the $N$-solitons solutions using the extended Moutard transformation and the Pfaffian expansion. Several examples are given. In Section 3, the $N$-solitonic wave function is derived using (7) and the Pfaffian expansion. Section 4 is used to prove a special property to characterize the $N$-solitons wave function. Section 5 is devoted to the concluding remarks.

\section{$2 \quad N$-solitons solutions}

In this section, one uses successive iterations of the extended Moutard transformation (4) to construct $N$-solitons solutions.

To obtain the $N$-solitons solutions, we assume that $V=0$ in (1) and recall that $\partial \bar{\partial}=\frac{1}{4} \triangle$. One considers $U=-\epsilon \neq 0$, i.e.,

$$
\partial \bar{\partial} \varphi=\epsilon \varphi, \quad \varphi_{t}=\varphi_{z z z}+\varphi_{\bar{z} \bar{z} \bar{z}}
$$

where $\epsilon$ is non-zero real constant. The general solution of (8) can be expressed as

$$
\varphi(z, \bar{z}, t)=\int_{\Gamma} e^{(i \lambda) z+(i \lambda)^{3} t+\frac{\epsilon}{i \lambda} \bar{z}+\frac{\epsilon^{3}}{(i \lambda)^{3}} t} \nu(\lambda) d \lambda
$$

where $\nu(\lambda)$ is an arbitrary distribution and $\Gamma$ is an arbitrary path of integration such that the r.h.s. of (9) is well defined.

Next, using (5) and (9), one can construct the $N$-solitons solutions. Let's take $\nu_{m}(\lambda)=$ $\delta\left(\lambda-p_{m}\right)$ and $\nu_{n}(\lambda)=a_{n} \delta\left(\lambda-q_{n}\right)$, where $p_{m}, a_{n}, q_{n}$ are complex numbers. Then one defines

$$
\phi_{m}=\frac{\varphi\left(p_{m}\right)}{\sqrt{3}}=\frac{1}{\sqrt{3}} e^{F\left(p_{m}\right)}, \quad \psi_{n}=a_{n} \frac{\varphi\left(q_{n}\right)}{\sqrt{3}}=\frac{a_{n}}{\sqrt{3}} e^{F\left(q_{n}\right)}
$$

where

$$
F(\lambda)=(i \lambda) z+(i \lambda)^{3} t+\frac{\epsilon}{i \lambda} \bar{z}+\frac{\epsilon^{3}}{(i \lambda)^{3}} t .
$$

Then a direct calculation of the extended Moutard transformation (4) can yield

$$
\begin{aligned}
W\left(\phi_{m}, \psi_{n}\right) & =i a_{n} \frac{q_{n}-p_{m}}{q_{n}+p_{m}} e^{F\left(p_{m}\right)+F\left(q_{n}\right)}, \quad W\left(\phi_{m}, \phi_{n}\right)=i \frac{p_{n}-p_{m}}{p_{n}+p_{m}} e^{F\left(p_{m}\right)+F\left(p_{n}\right)}, \\
W\left(\psi_{m}, \psi_{n}\right) & =i a_{m} a_{n} \frac{q_{n}-q_{m}}{q_{n}+q_{m}} e^{F\left(q_{m}\right)+F\left(q_{n}\right)} .
\end{aligned}
$$

The $N$-solitons solutions are defined by

$$
U(z, \bar{z}, t)=-\epsilon+2 \partial \bar{\partial} \ln \tau_{N}(z, \bar{z}, t), \quad V(z, \bar{z}, t)=2 \partial \partial \ln \tau_{N}(z, \bar{z}, t),
$$

and then

$$
U(z, \bar{z}, t) \rightarrow-\epsilon \quad \text { as } \quad z \bar{z} \rightarrow \infty
$$


where $t$ is fixed. The $\tau$-functions are defined as follows. For simplicity, let's denote

$$
W\left(p_{m}, q_{n}\right)=W\left(\phi_{m}, \psi_{n}\right), \quad W\left(p_{m}, p_{n}\right)=W\left(\phi_{m}, \phi_{n}\right), \quad W\left(q_{m}, q_{n}\right)=W\left(\psi_{m}, \psi_{n}\right),
$$

and notice that $F(-\lambda)=-F(\lambda)$. The $\tau_{N}$ is defined as

$$
\tau_{N}(z, \bar{z}, t)=\operatorname{Pf}\left(-p_{1}, q_{1},-p_{2}, q_{2},-p_{3}, q_{3}, \ldots,-p_{N}, q_{N}\right),
$$

where

$$
\begin{aligned}
& \left(-p_{m},-p_{n}\right)=W\left(-p_{m},-p_{n}\right), \quad\left(-p_{m}, q_{n}\right)=W\left(-p_{m}, q_{n}\right)+\delta_{m n}, \\
& \left(q_{m}, q_{n}\right)=W\left(q_{m}, q_{n}\right) .
\end{aligned}
$$

To get the expansion of (12), we use the following useful formula [14, 38]

$$
\operatorname{Pf}(\mathbf{A}+\mathbf{B})=\sum_{r=0}^{s} \sum_{\alpha \in I_{2 r}^{m}}(-1)^{|\alpha|-r} \operatorname{Pf}\left(\mathbf{A}_{\alpha}\right) \operatorname{Pf}\left(\mathbf{B}_{\alpha^{c}}\right),
$$

where $\mathbf{A}$ and $\mathbf{B}$ are $m \times m$ matrices and $s=[m / 2]$ is the integer part of $m / 2$; moreover, we denote by $\alpha^{c}$ the complementary set of $\alpha$ in the subset $\{1,2,3, \ldots, m\}$ which is arranged in increasing order, and $|\alpha|=\alpha_{1}+\alpha_{2}+\cdots+\alpha_{2 r}$ for $\alpha=\left(\alpha_{1}, \alpha_{2}, \ldots, \alpha_{2 r}\right)$. For the case (12), one has

$$
\mathbf{A}_{N}(z, \bar{z}, t)=\left[\begin{array}{cccccc}
0 & \left(-p_{1}, q_{1}\right) & \left(-p_{1},-p_{2}\right) & \left(-p_{1}, q_{2}\right) & \cdots & \left(-p_{1}, q_{N}\right) \\
\left(q_{1},-p_{1}\right) & 0 & \left(q_{1},-p_{2}\right) & \left(q_{1}, q_{2}\right) & \cdots & \left(q_{1}, q_{N}\right) \\
\left(-p_{2},-p_{1}\right) & \left(-p_{2}, q_{1}\right) & 0 & \left(-p_{2}, q_{2}\right) & \cdots & \left(-p_{2}, q_{N}\right) \\
\vdots & \vdots & \vdots & \ldots & \vdots & \\
\left(q_{N},-p_{1}\right) & \left(q_{N}, q_{1}\right) & \left(q_{N},-p_{2}\right) & \left(q_{N}, q_{2}\right) & \cdots & 0
\end{array}\right]
$$

and

$$
\mathbf{B}_{N}=\left[\begin{array}{cccccc}
0 & 1 & 0 & 0 & \cdots & 0 \\
-1 & 0 & 0 & 0 & \cdots & 0 \\
0 & 0 & 0 & 1 & \cdots & 0 \\
0 & 0 & -1 & 0 & \cdots & 0 \\
\vdots & \vdots & \vdots & \cdots & \vdots & \\
0 & 0 & 0 & 0 & \cdots & 1 \\
0 & 0 & 0 & \cdots & -1 & 0
\end{array}\right]
$$

where $\mathbf{A}_{N}$ and $\mathbf{B}_{N}$ are $2 N \times 2 N$ matrices. Hence by (14) one can have the expansion of (12), i.e.,

$$
\tau_{N}=1+\sum_{\ell=1}^{N} f_{\ell}+\sum_{m=2}\left(\sum_{1 \leq \ell_{1}<\ell_{2}<\cdots<\ell_{m} \leq N} f_{\ell_{1}} f_{\ell_{2}} \cdots f_{\ell_{m}} \prod_{1 \leq j<k \leq m} \mathbb{P}_{\ell_{j} \ell_{k}}\right),
$$

where

$$
f_{\ell}=i a_{\ell} \frac{p_{\ell}+q_{\ell}}{q_{\ell}-p_{\ell}} e^{F\left(q_{\ell}\right)-F\left(p_{\ell}\right)}, \quad \mathbb{P}_{\ell_{j} \ell_{k}}=\frac{\left(p_{\ell_{j}}-p_{\ell_{k}}\right)\left(q_{\ell_{j}}-q_{\ell_{k}}\right)\left(p_{\ell_{j}}+q_{\ell_{k}}\right)\left(q_{\ell_{j}}+p_{\ell_{k}}\right)}{\left(p_{\ell_{j}}+p_{\ell_{k}}\right)\left(q_{\ell_{j}}+q_{\ell_{k}}\right)\left(p_{\ell_{j}}-q_{\ell_{k}}\right)\left(q_{\ell_{j}}-p_{\ell_{k}}\right)}
$$

Here we have utilized the formula that if $\mathbf{C}$ is a $2 N \times 2 N$ matrix with $(i, j)$-th entry $\frac{\alpha_{i}-\alpha_{j}}{\alpha_{i}+\alpha_{j}}$, then one has the Schur identity [32, 36]

$$
\operatorname{Pf}(\mathbf{C})=\prod_{1 \leq i<j \leq 2 N}\left(\frac{\alpha_{i}-\alpha_{j}}{\alpha_{i}+\alpha_{j}}\right) .
$$


Next, we illustrate the formula (15) (or 12) with several examples.

(1) One-soliton solution:

$$
\mathbf{A}_{1}(z, \bar{z}, t)=\left[\begin{array}{cc}
0 & \left(-p_{1}, q_{1}\right) \\
\left(q_{1},-p_{1}\right) & 0
\end{array}\right], \quad \mathbf{B}_{1}=\left[\begin{array}{cc}
0 & 1 \\
-1 & 0
\end{array}\right] .
$$

Then

$$
\tau_{1}=\operatorname{Pf}\left(\mathbf{A}_{1}+\mathbf{B}_{1}\right)=1+i a_{1} \frac{q_{1}+p_{1}}{q_{1}-p_{1}} e^{F\left(q_{1}\right)-F\left(p_{1}\right)} .
$$

(2) Two-solitons solution:

$$
\begin{aligned}
& \mathbf{A}_{2}(z, \bar{z}, t)=\left[\begin{array}{cccc}
0 & \left(-p_{1}, q_{1}\right) & \left(-p_{1},-p_{2}\right) & \left(-p_{1}, q_{2}\right) \\
\left(q_{1},-p_{1}\right) & 0 & \left(q_{1},-p_{2}\right) & \left(q_{1}, q_{2}\right) \\
\left(-p_{2},-p_{1}\right) & \left(-p_{2}, q_{1}\right) & 0 & \left(-p_{2}, q_{2}\right) \\
\left(q_{2},-p_{1}\right) & \left(q_{2}, q_{1}\right) & \left(q_{2},-p_{2}\right) & 0
\end{array}\right], \\
& \mathbf{B}_{2}=\left[\begin{array}{cccc}
0 & 1 & 0 & 0 \\
-1 & 0 & 0 & 0 \\
0 & 0 & 0 & 1 \\
0 & 0 & -1 & 0
\end{array}\right] .
\end{aligned}
$$

Then

$$
\begin{aligned}
\tau_{2}= & \operatorname{Pf}\left(\mathbf{A}_{2}+\mathbf{B}_{2}\right)=1+i a_{1} \frac{p_{1}+q_{1}}{q_{1}-p_{1}} e^{F\left(q_{1}\right)-F\left(p_{1}\right)}+i a_{2} \frac{p_{2}+q_{2}}{q_{2}-p_{2}} e^{F\left(q_{2}\right)-F\left(p_{2}\right)} \\
& +i a_{1} i a_{2} \frac{p_{1}+q_{1}}{q_{1}-p_{1}} \frac{p_{2}+q_{2}}{q_{2}-p_{2}} \frac{p_{2}-p_{1}}{p_{2}+p_{1}} \frac{q_{2}-q_{1}}{q_{2}+q_{1}} \frac{p_{1}+q_{2}}{q_{2}-p_{1}} \frac{p_{2}+q_{1}}{p_{2}-q_{1}} e^{F\left(q_{1}\right)-F\left(p_{1}\right)+F\left(q_{2}\right)-F\left(p_{2}\right)}
\end{aligned}
$$

or

$$
\tau_{2}=1+f_{1}+f_{2}+\mathbb{P}_{12} f_{1} f_{2}
$$

where

$$
\begin{aligned}
& f_{1}=i a_{1} \frac{p_{1}+q_{1}}{q_{1}-p_{1}} e^{F\left(q_{1}\right)-F\left(p_{1}\right)}, \quad f_{2}=i a_{2} \frac{p_{2}+q_{2}}{q_{2}-p_{2}} e^{F\left(q_{2}\right)-F\left(p_{2}\right)}, \\
& \mathbb{P}_{12}=\frac{p_{1}-p_{2}}{p_{1}+p_{2}} \frac{q_{1}-q_{2}}{q_{1}+q_{2}} \frac{p_{1}+q_{2}}{p_{1}-q_{2}} \frac{q_{1}+p_{2}}{q_{1}-p_{2}} .
\end{aligned}
$$

The $\tau_{2}$ soliton (17) is also found in [2] using the $\bar{\partial}$-dressing method.

(3) Three-solitons solutin:

$$
\begin{aligned}
& \mathbf{A}_{3}(z, \bar{z}, t)=\left[\begin{array}{cccccc}
0 & \left(-p_{1}, q_{1}\right) & \left(-p_{1},-p_{2}\right) & \left(-p_{1}, q_{2}\right) & \left(-p_{1},-p_{3}\right) & \left(-p_{1}, q_{3}\right) \\
\left(q_{1},-p_{1}\right) & 0 & \left(q_{1},-p_{2}\right) & \left(q_{1}, q_{2}\right) & \left(q_{1},-p_{3}\right) & \left(q_{1}, q_{3}\right) \\
\left(-p_{2},-p_{1}\right) & \left(-p_{2}, q_{1}\right) & 0 & \left(-p_{2}, q_{2}\right) & \left(-p_{2},-p_{3}\right) & \left(-p_{2}, q_{3}\right) \\
\left(q_{2},-p_{1}\right) & \left(q_{2}, q_{1}\right) & \left(q_{2},-p_{2}\right) & 0 & \left(q_{2},-p_{3}\right) & \left(q_{2}, q_{3}\right) \\
\left(-p_{3},-p_{1}\right) & \left(-p_{3}, q_{1}\right) & \left(-p_{3},-p_{2}\right) & \left(-p_{3}, q_{2}\right) & 0 & \left(-p_{3}, q_{3}\right) \\
\left(q_{3},-p_{1}\right) & \left(q_{3}, q_{1}\right) & \left(q_{3},-p_{2}\right) & \left(q_{3}, q_{2}\right) & \left(q_{3},-p_{3}\right) & 0
\end{array}\right] \\
& \mathbf{B}_{3}=\left[\begin{array}{cccccc}
0 & 1 & 0 & 0 & 0 & 0 \\
-1 & 0 & 0 & 0 & 0 & 0 \\
0 & 0 & 0 & 1 & 0 & 0 \\
0 & 0 & -1 & 0 & 0 & 0 \\
0 & 0 & 0 & 0 & 0 & 1 \\
0 & 0 & 0 & 0 & -1 & 0
\end{array}\right]
\end{aligned}
$$


Then

$$
\begin{aligned}
\tau_{3} & =\operatorname{Pf}\left(\mathbf{A}_{3}+\mathbf{B}_{3}\right) \\
& =1+f_{1}+f_{2}+f_{3}+\mathbb{P}_{12} f_{1} f_{2}+\mathbb{P}_{13} f_{1} f_{3}+\mathbb{P}_{23} f_{2} f_{3}+\mathbb{P}_{12} \mathbb{P}_{13} \mathbb{P}_{23} f_{1} f_{2} f_{3},
\end{aligned}
$$

where

$$
\begin{aligned}
& f_{1}=i a_{1} \frac{p_{1}+q_{1}}{q_{1}-p_{1}} e^{F\left(q_{1}\right)-F\left(p_{1}\right)}, \quad f_{2}=i a_{2} \frac{p_{2}+q_{2}}{q_{2}-p_{2}} e^{F\left(q_{2}\right)-F\left(p_{2}\right)}, \\
& f_{3}=i a_{3} \frac{p_{3}+q_{3}}{q_{3}-p_{3}} e^{F\left(q_{3}\right)-F\left(p_{3}\right)}, \quad \mathbb{P}_{12}=\frac{p_{1}-p_{2}}{p_{1}+p_{2}} \frac{q_{1}-q_{2}}{q_{1}+q_{2}} \frac{p_{1}+q_{2}}{p_{1}-q_{2}} \frac{q_{1}+p_{2}}{q_{1}-p_{2}}, \\
& \mathbb{P}_{13}=\frac{p_{1}+q_{3}}{p_{1}-q_{3}} \frac{q_{1}+p_{3}}{q_{1}-p_{3}} \frac{p_{1}-p_{3}}{p_{1}+p_{3}} \frac{q_{1}-q_{3}}{q_{1}+q_{3}}, \quad \mathbb{P}_{23}=\frac{p_{2}+q_{3}}{p_{2}-q_{3}} \frac{q_{2}+p_{3}}{q_{2}-p_{3}} \frac{p_{2}-p_{3}}{p_{2}+p_{3}} \frac{q_{2}-q_{3}}{q_{2}+q_{3}} .
\end{aligned}
$$

\section{The wave functions}

In this section, one uses (7) to construct the corresponding wave function of the $\tau$ function (15).

From (7), one knows that the corresponding wave function of the $N$-solitons (15) can be written as

$$
\varphi_{N}=\frac{P\left(\frac{\varphi\left(-p_{1}\right)}{\sqrt{3}}, \frac{\varphi\left(q_{1}\right)}{\sqrt{3}}, \frac{\varphi\left(-p_{2}\right)}{\sqrt{3}}, \frac{\varphi\left(q_{2}\right)}{\sqrt{3}}, \ldots, \frac{\varphi\left(-p_{N}\right)}{\sqrt{3}}, \frac{\varphi\left(q_{N}\right)}{\sqrt{3}}, \frac{\varphi(\lambda)}{\sqrt{3}}\right)}{\tau_{N}} .
$$

Using the notations in (11), (12) and (13), we can express $\varphi_{N}$ as

$$
\varphi_{N}=\frac{P\left(-p_{1}, q_{1},-p_{2}, q_{2}, \ldots,-p_{N}, q_{N}, \lambda\right)}{\tau_{N}} .
$$

But we notice that

$$
\left(-p_{m}, \lambda\right)^{\sharp}=W\left(-p_{m}, \lambda\right), \quad\left(q_{m}, \lambda\right)^{\sharp}=W\left(q_{m}, \lambda\right),
$$

where $(\cdot)^{\sharp}$ means there is no $\delta_{m n}$ here when compared with (13). Now, let's compute $P\left(-p_{1}, q_{1}\right.$, $\left.-p_{2}, q_{2}, \ldots,-p_{N}, q_{N}, \lambda\right)$ using (14). In this case,

$$
P\left(-p_{1}, q_{1},-p_{2}, q_{2}, \ldots,-p_{N}, q_{N}, \lambda\right)=\operatorname{Pf}\left(M_{N}+Q_{N}\right),
$$

where

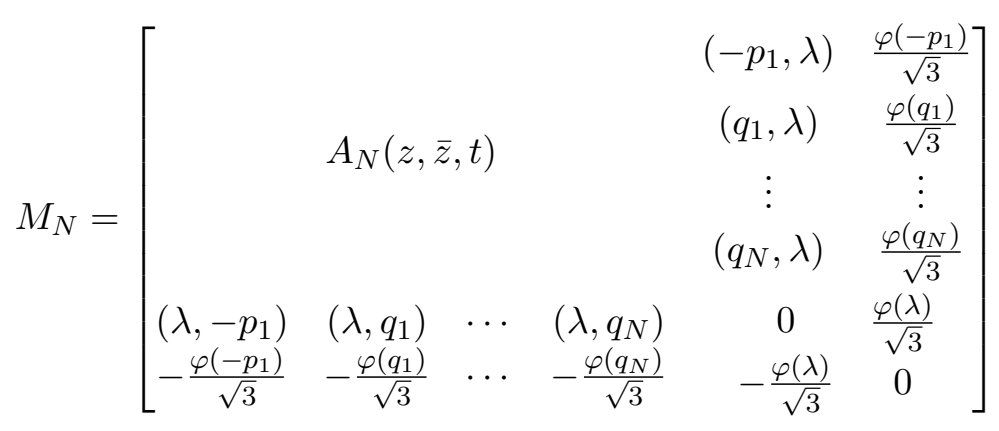

and

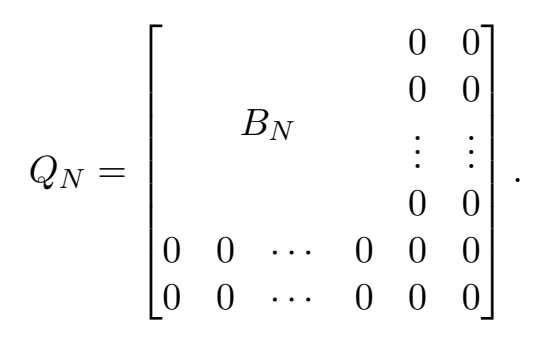


Using (16), a simple calculation yields

$$
\begin{aligned}
& \operatorname{Pf}\left(M_{N}+Q_{N}\right) \\
& \quad=\phi\left[1+\sum_{\ell=1}^{N} h_{\ell}(\lambda)+\sum_{m=2}^{N}\left(\sum_{1 \leqq \ell_{1}<\ell_{2}<\ell_{3}<\cdots<\ell_{m} \leqq N} h_{\ell_{1}} h_{\ell_{2}} \cdots h_{\ell_{m}} \prod_{1 \leqq j<k \leqq m} \mathbb{P}_{\ell_{j} \ell_{k}}\right)\right] \\
& =\phi \hat{\chi}_{N}(\lambda),
\end{aligned}
$$

where

$$
\begin{aligned}
& \phi=\frac{\varphi(\lambda)}{\sqrt{3}}, \quad h_{\ell}(\lambda)=i a_{\ell} \frac{p_{\ell}+q_{\ell}}{q_{\ell}-p_{\ell}} \frac{p_{\ell}+\lambda}{p_{\ell}-\lambda} \frac{q_{\ell}-\lambda}{q_{\ell}+\lambda} e^{F\left(q_{\ell}\right)-F\left(p_{\ell}\right)}=f_{\ell} \frac{p_{\ell}+\lambda}{p_{\ell}-\lambda} \frac{q_{\ell}-\lambda}{q_{\ell}+\lambda}, \\
& \hat{\chi}_{N}(\lambda)=1+\sum_{\ell=1}^{N} h_{\ell}(\lambda)+\sum_{m=2}^{N}\left(\sum_{1 \leqq \ell_{1}<\ell_{2}<\ell_{3}<\cdots<\ell_{m} \leqq N} h_{\ell_{1}} h_{\ell_{2}} \cdots h_{\ell_{m}} \prod_{1 \leqq j<k \leqq m} \mathbb{P}_{\ell_{j} \ell_{k}}\right),
\end{aligned}
$$

and $\mathbb{P}_{\ell_{j} \ell_{k}}$ is defined in (15).

We give several examples here.

(1) The one-soliton wave function:

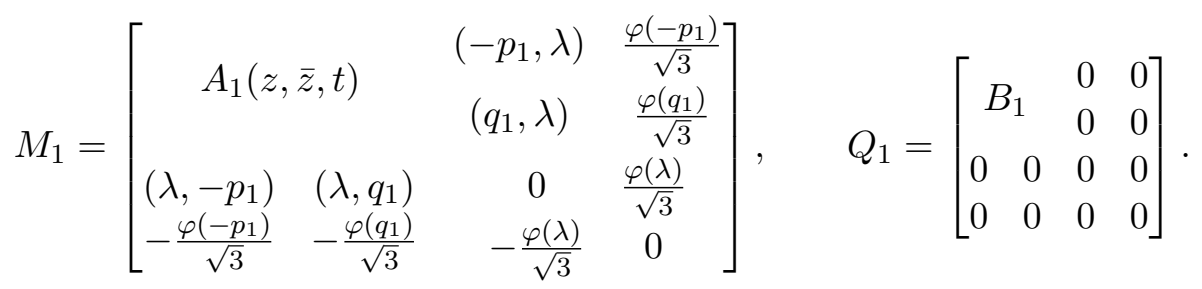

Then

$$
\varphi_{1}=\frac{P\left(-p_{1}, q_{1}, \lambda\right)}{\tau_{1}}=\frac{\operatorname{Pf}\left(M_{1}+Q_{1}\right)}{\tau_{1}}=\frac{\phi}{\tau_{1}}\left(1+i a_{1} \frac{p_{1}+q_{1}}{q_{1}-p_{1}} \frac{p_{1}+\lambda}{p_{1}-\lambda} \frac{q_{1}-\lambda}{q_{1}+\lambda} e^{F\left(q_{1}\right)-F\left(p_{1}\right)}\right) .
$$

We remark that

$$
\begin{aligned}
\varphi_{1} & =\frac{\phi}{1+f_{1}}\left[1+i a_{1} \frac{p_{1}+\lambda}{p_{1}-\lambda} \frac{q_{1}-\lambda}{q_{1}+\lambda} f_{1}\right]=\frac{\phi}{1+f_{1}}\left[1+i a_{1}\left(\frac{2 p_{1}}{p_{1}-\lambda}-1\right)\left(\frac{2 q_{1}}{q_{1}+\lambda}-1\right) f_{1}\right] \\
& =\phi\left[\frac{\left(1+f_{1}\right)+2 i a_{1}\left(\frac{p_{1}}{p_{1}-\lambda}-\frac{q_{1}}{q_{1}+\lambda}\right) e^{F\left(q_{1}\right)-F\left(p_{1}\right)}}{1+f_{1}}\right] \\
& =\phi\left[1-2 i a_{1}\left(\frac{p_{1}}{\lambda-p_{1}}+\frac{q_{1}}{\lambda+q_{1}}\right) \frac{e^{F\left(q_{1}\right)-F\left(p_{1}\right)}}{\tau_{1}}\right] .
\end{aligned}
$$

This is the one-soliton wave function in $[2$, p. 9].

(2) The two-soliton wave function:

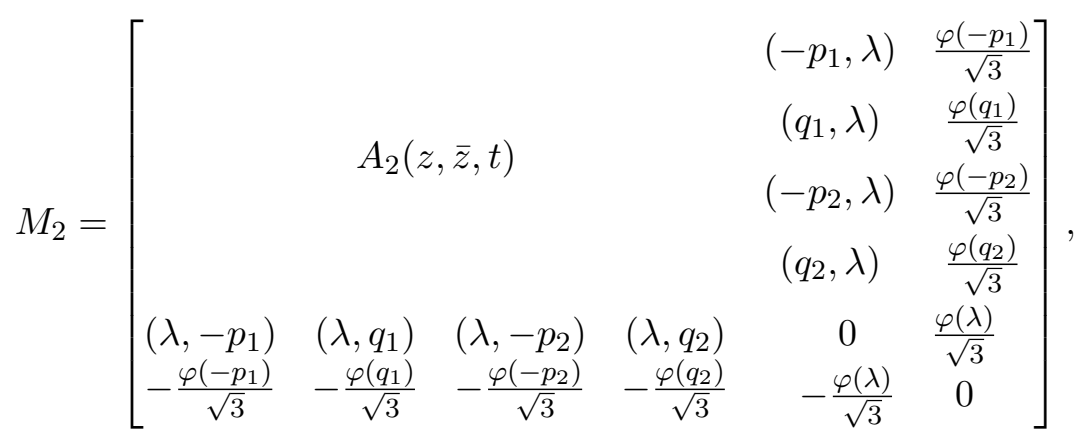




$$
Q_{2}=\left[\begin{array}{llllll} 
& & & & 0 & 0 \\
& B_{2} & & 0 & 0 \\
& & & & 0 & 0 \\
0 & 0 & 0 & 0 & 0 & 0 \\
0 & 0 & 0 & 0 & 0 & 0
\end{array}\right] .
$$

Then using (21), one has

$$
\begin{aligned}
\varphi_{2}= & \frac{P\left(-p_{1}, q_{1},-p_{2}, q_{2}, \lambda\right)}{\tau_{2}}=\frac{\operatorname{Pf}\left(M_{2}+Q_{2}\right)}{\tau_{2}}=\frac{\phi}{\tau_{2}}\left[1+h_{1}(\lambda)+h_{2}(\lambda)+\mathbb{P}_{12} h_{1}(\lambda) h_{2}(\lambda)\right] \\
= & \frac{\phi}{\tau_{2}}\left(1+i a_{1} \frac{p_{1}+q_{1}}{q_{1}-p_{1}} \frac{p_{1}+\lambda}{p_{1}-\lambda} \frac{q_{1}-\lambda}{q_{1}+\lambda} e^{F\left(q_{1}\right)-F\left(p_{1}\right)}+i a_{2} \frac{p_{2}+q_{2}}{q_{2}-p_{2}} \frac{p_{2}+\lambda}{p_{2}-\lambda} \frac{q_{2}-\lambda}{q_{2}+\lambda} e^{F\left(q_{2}\right)-F\left(p_{2}\right)}\right. \\
& \left.+i a_{1} i a_{2} \frac{p_{1}+q_{1}}{q_{1}-p_{1}} \frac{p_{1}+\lambda}{p_{1}-\lambda} \frac{q_{1}-\lambda}{q_{1}+\lambda} \frac{p_{2}+q_{2}}{q_{2}-p_{2}} \frac{p_{2}+\lambda}{p_{2}-\lambda} \frac{q_{2}-\lambda}{q_{2}+\lambda} e^{F\left(q_{1}\right)+F\left(q_{2}\right)-F\left(p_{1}\right)-F\left(p_{2}\right)}\right) .
\end{aligned}
$$

(3) Three-solitons wave function:

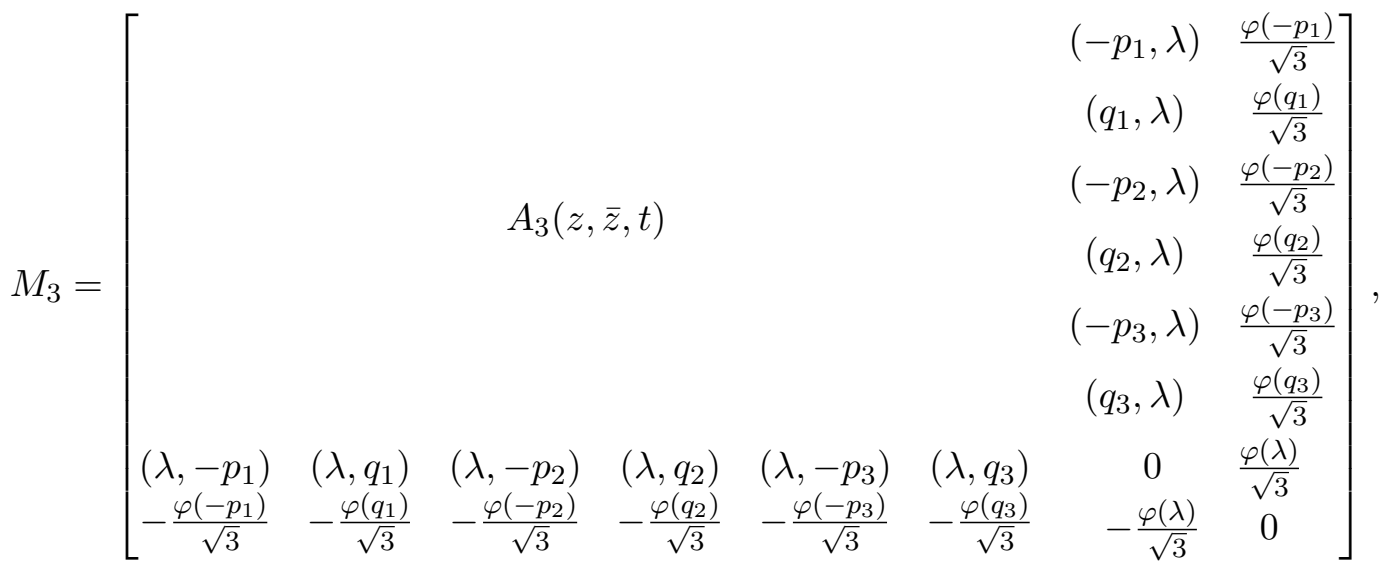

$$
\begin{aligned}
& Q_{3}=\left[\begin{array}{llllllll} 
& & & & & & 0 & 0 \\
& & & & & & 0 & 0 \\
& & B_{3} & & & 0 & 0 \\
& & & & & 0 & 0 \\
& & & & & & 0 & 0 \\
& & & & & & 0 & 0 \\
0 & 0 & 0 & 0 & 0 & 0 & 0 & 0 \\
0 & 0 & 0 & 0 & 0 & 0 & 0 & 0
\end{array}\right] .
\end{aligned}
$$

From (21), we get

$$
\begin{aligned}
\varphi_{3}= & \frac{P\left(-p_{1}, q_{1},-p_{2}, q_{2},-p_{3}, q_{3}, \lambda\right)}{\tau_{3}}=\frac{\operatorname{Pf}\left(M_{3}+Q_{3}\right)}{\tau_{3}} \\
= & \frac{\phi}{\tau_{3}}\left[1+h_{1}(\lambda)+h_{2}(\lambda)+h_{3}(\lambda)+\mathbb{P}_{12} h_{1}(\lambda) h_{2}(\lambda)+\mathbb{P}_{13} h_{1}(\lambda) h_{3}(\lambda)+\mathbb{P}_{23} h_{2}(\lambda) h_{3}(\lambda)\right. \\
& \left.+\mathbb{P}_{12} \mathbb{P}_{13} \mathbb{P}_{23} h_{1}(\lambda) h_{2}(\lambda) h_{3}(\lambda)\right],
\end{aligned}
$$

where

$$
\begin{aligned}
& h_{1}=i a_{1} \frac{p_{1}+\lambda}{p_{1}-\lambda} \frac{q_{1}-\lambda}{q_{1}+\lambda} \frac{p_{1}+q_{1}}{q_{1}-p_{1}} e^{F\left(q_{1}\right)-F\left(p_{1}\right)}, \quad h_{2}=i a_{2} \frac{p_{2}+\lambda}{p_{2}-\lambda} \frac{q_{2}-\lambda}{q_{2}+\lambda} \frac{p_{2}+q_{2}}{q_{2}-p_{2}} e^{F\left(q_{2}\right)-F\left(p_{2}\right)}, \\
& h_{3}=i a_{3} \frac{p_{3}+\lambda}{p_{3}-\lambda} \frac{q_{3}-\lambda}{q_{3}+\lambda} \frac{p_{3}+q_{3}}{q_{3}-p_{3}} e^{F\left(q_{3}\right)-F\left(p_{3}\right)}
\end{aligned}
$$

and $\mathbb{P}_{12}, \mathbb{P}_{13}$ and $\mathbb{P}_{23}$ are defined in (18). 


\section{A property of $N$-solitons wave function}

In this section, we will express the wave function (19) as another form to generalize the equation (22) to $N$-solitons case.

Firstly, according to the Pfaffian expansion in [11], it is not difficult to see that

$$
\begin{aligned}
& \widetilde{\operatorname{Pf}}\left(b_{1}, b_{2}, b_{3}, b_{4}, \ldots, b_{2 n-1}, b_{2 n}, b_{2 n+1}\right) \\
& \quad=\sum_{m=1}^{2 n+1}(-1)^{j+m}\left(b_{j}, b_{m}\right) \widetilde{\operatorname{Pf}}\left(b_{1}, b_{2}, \ldots, \hat{b}_{j}, \ldots, \hat{b}_{m}, \ldots, b_{2 n}, b_{2 n+1}\right), \\
& \quad \text { for } \quad j=1,2, \ldots, 2 n+1,
\end{aligned}
$$

where $\hat{b}_{j}$ and $\hat{b}_{m}$ mean these two terms are omitted.

Secondly, noticing (10) and letting $\lambda=-p_{\alpha}$ or $\lambda=q_{\alpha}, \alpha=1,2, \ldots, n$, we have

$$
\begin{aligned}
& \widetilde{\operatorname{Pf}}\left(-p_{1}, q_{1},-p_{2}, q_{2}, \ldots,-p_{\alpha}, q_{\alpha},-p_{\alpha+1}, q_{\alpha+1}, \ldots,-p_{N}, q_{N},-p_{\alpha}\right) \\
& \quad=\widetilde{\operatorname{Pf}}\left(-p_{1}, q_{1},-p_{2}, q_{2}, \ldots,-p_{\alpha},-p_{\alpha+1}, q_{\alpha+1}, \ldots,-p_{N}, q_{N}\right)=\phi\left(-p_{\alpha}\right) \hat{\chi}_{N}\left(-p_{\alpha}\right), \\
& \widetilde{\operatorname{Pf}}\left(-p_{1}, q_{1},-p_{2}, q_{2}, \ldots, q_{\alpha-1},-p_{\alpha}, q_{\alpha},-p_{\alpha+1}, q_{\alpha+1}, \ldots,-p_{N}, q_{N}, q_{\alpha}\right) \\
& \quad=\widetilde{\operatorname{Pf}}\left(-p_{1}, q_{1},-p_{2}, q_{2}, \ldots, q_{\alpha-1}, q_{\alpha},-p_{\alpha+1}, q_{\alpha+1}, \ldots,-p_{N}, q_{N}\right)=a_{\alpha} \phi\left(q_{\alpha}\right) \hat{\chi}_{N}\left(q_{\alpha}\right) .
\end{aligned}
$$

They can be seen as follows. By (23), one has

$$
\begin{aligned}
\widetilde{\operatorname{Pf}} & \left(-p_{1}, q_{1},-p_{2}, q_{2}, \ldots,-p_{\alpha}, q_{\alpha},-p_{\alpha+1}, q_{\alpha+1}, \ldots,-p_{N}, q_{N},-p_{\alpha}\right) \\
= & -\left(q_{\alpha},-p_{1}\right) \widetilde{\operatorname{Pf}}\left(q_{1},-p_{2}, q_{2}, \ldots,-p_{\alpha-1}, q_{\alpha-1},-p_{\alpha},-p_{\alpha+1}, \ldots,-p_{N}, q_{N},-p_{\alpha}\right) \\
& +\left(q_{\alpha}, q_{1}\right) \widetilde{\operatorname{Pf}}\left(-p_{1},-p_{2}, q_{2}, \ldots,-p_{\alpha-1}, q_{\alpha-1},-p_{\alpha},-p_{\alpha+1}, \ldots,-p_{N}, q_{N},-p_{\alpha}\right)-\cdots \\
& -\left(q_{\alpha},-p_{\alpha}\right) \widetilde{\operatorname{Pf}}\left(-p_{1}, q_{1},-p_{2}, q_{2}, \ldots,-p_{\alpha-1}, q_{\alpha-1},-p_{\alpha+1}, \ldots,-p_{N}, q_{N},-p_{\alpha}\right)+\cdots \\
& +\left(q_{\alpha},-p_{\alpha}\right)^{\sharp} \widetilde{\operatorname{Pf}}\left(-p_{1}, q_{1},-p_{2}, q_{2}, \ldots,-p_{\alpha-1}, q_{\alpha-1},-p_{\alpha},-p_{\alpha+1}, \ldots,-p_{N}, q_{N}\right) \\
= & {\left[-\left(q_{\alpha},-p_{\alpha}\right)+\left(q_{\alpha},-p_{\alpha}\right)^{\sharp} \widetilde{\operatorname{Pf}}\left(-p_{1}, q_{1},-p_{2}, q_{2}, \ldots,-p_{\alpha-1}, q_{\alpha-1},-p_{\alpha},-p_{\alpha+1}, \ldots,-p_{N}, q_{N}\right)\right.} \\
= & \widetilde{\operatorname{Pf}}\left(-p_{1}, q_{1},-p_{2}, q_{2}, \ldots,-p_{\alpha-1}, q_{\alpha-1},-p_{\alpha},-p_{\alpha+1}, q_{\alpha+1}, \ldots,-p_{N}, q_{N}\right),
\end{aligned}
$$

where $\left(q_{\alpha},-p_{\alpha}\right)^{\sharp}$ is defined in $(20)$ and we know that

$$
\widetilde{\operatorname{Pf}}\left(\ldots,-p_{\alpha}, \ldots,-p_{\alpha}\right)=0 .
$$

The second equation of (24) can be proved similarly.

Finally, from (23), one yields

$$
\begin{aligned}
\widetilde{\operatorname{Pf}} & \left.-p_{1}, q_{1},-p_{2}, q_{2}, \ldots,-p_{N}, q_{N}, \lambda\right)=\left(\lambda,-p_{1}\right) \widetilde{\operatorname{Pf}}\left(q_{1},-p_{2}, q_{2}, \ldots,-p_{N}, q_{N}\right) \\
& -\left(\lambda, q_{1}\right) \widetilde{\operatorname{Pf}}\left(-p_{1},-p_{2}, q_{2}, \ldots,-p_{N}, q_{N}\right)+\left(\lambda,-p_{2}\right) \widetilde{\operatorname{Pf}}\left(q_{1},-p_{1}, q_{2}, \ldots,-p_{N}, q_{N}\right) \\
& -\left(\lambda, q_{2}\right) \widetilde{\operatorname{Pf}}\left(q_{1},-p_{1},-p_{2}, \ldots,-p_{N}, q_{N}\right)+\cdots \\
& +\left(\lambda,-p_{N}\right) \widetilde{\operatorname{Pf}}\left(-p_{1},-p_{2}, q_{2}, \ldots,-p_{N-1}, q_{N-1}, q_{N}\right) \\
& -\left(\lambda, q_{N}\right) \widetilde{\operatorname{Pf}}\left(-p_{1},-p_{2}, q_{2}, \ldots,-p_{N-1}, q_{N-1},-p_{N}\right)+\phi(\lambda) \tau_{N} .
\end{aligned}
$$

Also, the wave function (21) can be written as

$$
\varphi_{N}(\lambda)=\phi(\lambda) \chi_{N}(\lambda)
$$


where $\chi_{N}(\lambda)=\frac{\hat{\chi}_{N}(\lambda)}{\tau_{N}}$. Therefore, using $(24)$ and letting $\Delta F_{n}=F\left(q_{n}\right)-F\left(p_{n}\right)$, we get

$$
\begin{aligned}
\chi_{N}(\lambda)= & -\frac{p_{1}+\lambda}{\lambda-p_{1}} i a_{1} e^{\Delta F_{1}} \chi_{N}\left(q_{1}\right)-\frac{q_{1}-\lambda}{q_{1}+\lambda} i a_{1} e^{\Delta F_{1}} \chi_{N}\left(-p_{1}\right) \\
& -\frac{p_{2}+\lambda}{\lambda-p_{2}} i a_{2} e^{\Delta F_{2}} \chi_{N}\left(q_{2}\right)-\frac{q_{2}-\lambda}{q_{2}+\lambda} i a_{2} e^{\Delta F_{2}} \chi_{N}\left(-p_{2}\right)-\cdots \\
& -\frac{p_{N}+\lambda}{\lambda-p_{N}} i a_{N} e^{\Delta F_{N}} \chi_{N}\left(q_{N}\right)-\frac{q_{N}-\lambda}{q_{N}+\lambda} i a_{N} e^{\Delta F_{N}} \chi_{N}\left(-p_{N}\right) \\
= & 1-\frac{2 p_{1}}{\lambda-p_{1}} i a_{1} e^{\Delta F_{1}} \chi_{N}\left(q_{1}\right)-\frac{2 q_{1}}{q_{1}+\lambda} i a_{1} e^{\Delta F_{1}} \chi_{N}\left(-p_{1}\right) \\
& -\frac{2 p_{2}}{\lambda-p_{2}} i a_{2} e^{\Delta F_{2}} \chi_{N}\left(q_{2}\right)-\frac{2 q_{2}}{q_{2}+\lambda} i a_{2} e^{\Delta F_{2}} \chi_{N}\left(-p_{2}\right)-\cdots \\
& -\frac{2 p_{N}}{\lambda-p_{N}} i a_{N} e^{\Delta F_{N}} \chi_{N}\left(q_{N}\right)-\frac{2 q_{N}}{q_{N}+\lambda} i a_{N} e^{\Delta F_{N}} \chi_{N}\left(-p_{N}\right) \\
& +\left[-i a_{1} e^{\Delta F_{1}} \chi_{N}\left(q_{1}\right)+i a_{1} e^{\Delta F_{1}} \chi_{N}\left(-p_{1}\right)-i a_{2} e^{\Delta F_{2}} \chi_{N}\left(q_{2}\right)\right. \\
& \left.+i a_{2} e^{\Delta F_{2}} \chi_{N}\left(-p_{2}\right)-\cdots-i a_{N} e^{\Delta F_{N}} \chi_{N}\left(q_{N}\right)+i a_{N} e^{\Delta F_{N}} \chi_{N}\left(-p_{N}\right)\right] .
\end{aligned}
$$

Since $\widetilde{\operatorname{Pf}}\left(-p_{1}, q_{1},-p_{2}, q_{2}, \ldots,-p_{N}, q_{N}, 0\right)=\frac{\tau_{N}}{\sqrt{3}}$, we have $\hat{\chi}_{N}(0)=\tau_{N}$. Then the last term in $[\cdots]$ of $(25)$ is zero (or $\lim _{\lambda \rightarrow \infty} \chi_{N}(\lambda)=1$ ). Hence one has

$$
\begin{aligned}
\chi_{N}(\lambda)= & 1-\frac{2 p_{1}}{\lambda-p_{1}} i a_{1} e^{\Delta F_{1}} \chi_{N}\left(q_{1}\right)-\frac{2 q_{1}}{q_{1}+\lambda} i a_{1} e^{\Delta F_{1}} \chi_{N}\left(-p_{1}\right) \\
& -\frac{2 p_{2}}{\lambda-p_{2}} i a_{2} e^{\Delta F_{2}} \chi_{N}\left(q_{2}\right)-\frac{2 q_{2}}{q_{2}+\lambda} i a_{2} e^{\Delta F_{2}} \chi_{N}\left(-p_{2}\right)-\cdots \\
& -\frac{2 p_{N}}{\lambda-p_{N}} i a_{N} e^{\Delta F_{N}} \chi_{N}\left(q_{N}\right)-\frac{2 q_{N}}{q_{N}+\lambda} i a_{N} e^{\Delta F_{N}} \chi_{N}\left(-p_{N}\right) .
\end{aligned}
$$

This formula is also obtained by the d-bar dressing method when the d-bar data is the degenerate delta kernel [2, p. 6].

When $n=1$, we have (22). For $n=2$, from (21), one knows that

$$
\begin{aligned}
& \chi_{2}\left(-p_{1}\right)=\left[1+i a_{2} \frac{p_{2}+q_{2}}{q_{2}-p_{2}} \frac{p_{2}-p_{1}}{p_{2}+p_{1}} \frac{q_{2}+p_{1}}{q_{2}-p_{1}} e^{\triangle F_{2}}\right] / \tau_{2}, \\
& \chi_{2}\left(q_{1}\right)=\left[1+i a_{2} \frac{p_{2}+q_{2}}{q_{2}-p_{2}} \frac{p_{2}+q_{1}}{p_{2}-q_{1}} \frac{q_{2}-q_{1}}{q_{2}+q_{1}} e^{\triangle F_{2}}\right] / \tau_{2}, \\
& \chi_{2}\left(-p_{2}\right)=\left[1+i a_{1} \frac{p_{1}+q_{1}}{q_{1}-p_{1}} \frac{p_{1}-p_{2}}{p_{1}+p_{2}} \frac{q_{1}+p_{2}}{q_{1}-p_{2}} e^{\triangle F_{1}}\right] / \tau_{2}, \\
& \chi_{2}\left(q_{2}\right)=\left[1+i a_{1} \frac{p_{1}+q_{1}}{q_{1}-p_{1}} \frac{p_{1}+q_{2}}{p_{1}-q_{2}} \frac{q_{1}-q_{2}}{q_{1}+q_{2}} e^{\triangle F_{1}}\right] / \tau_{2} .
\end{aligned}
$$

Then

$$
\begin{aligned}
\chi_{2}(\lambda)= & 1-\frac{2 p_{1}}{\lambda-p_{1}} i a_{1} e^{\Delta F_{1}} \chi_{2}\left(q_{1}\right)-\frac{2 q_{1}}{q_{1}+\lambda} i a_{1} e^{\Delta F_{1}} \chi_{2}\left(-p_{1}\right) \\
& -\frac{2 p_{2}}{\lambda-p_{2}} i a_{2} e^{\Delta F_{2}} \chi_{2}\left(q_{2}\right)-\frac{2 q_{2}}{q_{2}+\lambda} i a_{2} e^{\Delta F_{2}} \chi_{2}\left(-p_{2}\right) .
\end{aligned}
$$

We remark that this formula also appears in [2, p. 10], the parameters being different. For $n=3$, by (21), we obtain

$$
\chi_{3}\left(-p_{1}\right)=\left[1+i a_{2} \frac{p_{2}+q_{2}}{q_{2}-p_{2}} \frac{p_{2}-p_{1}}{p_{2}+p_{1}} \frac{q_{2}+p_{1}}{q_{2}-p_{1}} e^{\triangle F_{2}}+i a_{3} \frac{p_{3}+q_{3}}{q_{3}-p_{3}} \frac{p_{3}-p_{1}}{p_{3}+p_{1}} \frac{q_{3}+p_{1}}{q_{3}-p_{1}} e^{\triangle F_{3}}\right.
$$




$$
\begin{aligned}
& \left.+i a_{2} i a_{3} \frac{p_{2}+q_{2}}{q_{2}-p_{2}} \frac{p_{2}-p_{1}}{p_{2}+p_{1}} \frac{q_{2}+p_{1}}{q_{2}-p_{1}} \frac{p_{3}+q_{3}}{q_{3}-p_{3}} \frac{p_{3}-p_{1}}{p_{3}+p_{1}} \frac{q_{3}+p_{1}}{q_{3}-p_{1}} \mathbb{P}_{23} e^{\triangle F_{2}+\triangle F_{3}}\right] / \tau_{3}, \\
& \chi_{3}\left(q_{1}\right)=\left[1+i a_{2} \frac{p_{2}+q_{2}}{q_{2}-p_{2}} \frac{p_{2}+q_{1}}{p_{2}-q_{1}} \frac{q_{2}-q_{1}}{q_{2}+q_{1}} e^{\triangle F_{2}}+i a_{3} \frac{p_{3}+q_{3}}{q_{3}-p_{3}} \frac{p_{2}+q_{1}}{p_{2}-q_{1}} \frac{q_{3}-q_{1}}{q_{3}+q_{1}} e^{\triangle F_{3}}\right. \\
& \left.+i a_{2} i a_{3} \frac{p_{2}+q_{2}}{q_{2}-p_{2}} \frac{p_{2}+q_{1}}{p_{2}-q_{1}} \frac{q_{2}-q_{1}}{q_{2}+q_{1}} \frac{p_{3}+q_{3}}{q_{3}-p_{3}} \frac{p_{2}+q_{1}}{p_{2}-q_{1}} \frac{q_{3}-q_{1}}{q_{3}+q_{1}} \mathbb{P}_{23} e^{\triangle F_{2}+\triangle F_{3}}\right] / \tau_{3}, \\
& \chi_{3}\left(-p_{2}\right)=\left[1+i a_{1} \frac{p_{1}+q_{1}}{q_{1}-p_{1}} \frac{p_{1}-p_{2}}{p_{1}+p_{2}} \frac{q_{1}+p_{2}}{q_{1}-p_{2}} e^{\triangle F_{1}}+i a_{3} \frac{p_{3}+q_{3}}{p_{3}-q_{3}} \frac{p_{3}-p_{2}}{p_{3}+p_{2}} \frac{q_{3}+p_{2}}{q_{3}-p_{2}} e^{\triangle F_{3}}\right. \\
& \left.+i a_{1} i a_{3} \frac{p_{1}+q_{1}}{q_{1}-p_{1}} \frac{p_{1}-p_{2}}{p_{1}+p_{2}} \frac{q_{1}+p_{2}}{q_{1}-p_{2}} \frac{p_{3}+q_{3}}{p_{3}-q_{3}} \frac{p_{3}-p_{2}}{p_{3}+p_{2}} \frac{q_{3}+p_{2}}{q_{3}-p_{2}} \mathbb{P}_{13} e^{\triangle F_{1}+\triangle F_{3}}\right] / \tau_{3}, \\
& \chi_{3}\left(q_{2}\right)=\left[1+i a_{1} \frac{p_{1}+q_{1}}{q_{1}-p_{1}} \frac{p_{1}+q_{2}}{p_{1}-q_{2}} \frac{q_{1}-q_{2}}{q_{1}+q_{2}} e^{\triangle F_{1}}+i a_{3} \frac{p_{3}+q_{3}}{q_{3}-p_{3}} \frac{p_{3}+q_{2}}{p_{3}-q_{2}} \frac{q_{3}-q_{2}}{q_{3}+q_{2}} e^{\triangle F_{3}}\right. \\
& \left.+i a_{1} i a_{3} \frac{p_{1}+q_{1}}{q_{1}-p_{1}} \frac{p_{1}+q_{2}}{p_{1}-q_{2}} \frac{q_{1}-q_{2}}{q_{1}+q_{2}} \frac{p_{3}+q_{3}}{q_{3}-p_{3}} \frac{p_{3}+q_{2}}{p_{3}-q_{2}} \frac{q_{3}-q_{2}}{q_{3}+q_{2}} \mathbb{P}_{13} e^{\triangle F_{1}+\triangle F_{3}}\right] / \tau_{3}, \\
& \chi_{3}\left(-p_{3}\right)=\left[1+i a_{1} \frac{p_{1}+q_{1}}{p_{1}-q_{1}} \frac{p_{1}-p_{3}}{p_{1}+p_{3}} \frac{q_{1}+p_{3}}{q_{1}-p_{3}} e^{\triangle F_{1}}+i a_{2} \frac{p_{2}+q_{2}}{q_{2}-p_{2}} \frac{p_{2}-p_{3}}{p_{2}+p_{3}} \frac{q_{2}+p_{3}}{q_{2}-p_{3}} e^{\triangle F_{2}}\right. \\
& \left.+i a_{1} i a_{2} \frac{p_{1}+q_{1}}{p_{1}-q_{1}} \frac{p_{1}-p_{3}}{p_{1}+p_{3}} \frac{q_{1}+p_{3}}{q_{1}-p_{3}} \frac{p_{2}+q_{2}}{q_{2}-p_{2}} \frac{p_{2}-p_{3}}{p_{2}+p_{3}} \frac{q_{2}+p_{3}}{q_{2}-p_{3}} \mathbb{P}_{12} e^{\triangle F_{1}+\triangle F_{2}}\right] / \tau_{3}, \\
& \chi_{3}\left(q_{3}\right)=\left[1+i a_{1} \frac{p_{1}+q_{1}}{q_{1}-p_{1}} \frac{p_{1}+q_{3}}{p_{1}-q_{3}} \frac{q_{1}-q_{3}}{q_{1}+q_{3}} e^{\triangle F_{1}}+i a_{2} \frac{p_{2}+q_{2}}{q_{2}-p_{2}} \frac{p_{2}+q_{3}}{p_{2}-q_{3}} \frac{q_{2}-q_{3}}{q_{2}+q_{3}} e^{\triangle F_{2}}\right. \\
& \left.+i a_{1} i a_{2} \frac{p_{1}+q_{1}}{q_{1}-p_{1}} \frac{p_{1}+q_{3}}{p_{1}-q_{3}} \frac{q_{1}-q_{3}}{q_{1}+q_{3}} \frac{p_{2}+q_{2}}{q_{2}-p_{2}} \frac{p_{2}+q_{3}}{p_{2}-q_{3}} \frac{q_{2}-q_{3}}{q_{2}+q_{3}} \mathbb{P}_{12} e^{\triangle F_{1}+\triangle F_{2}}\right] / \tau_{3},
\end{aligned}
$$

where $\mathbb{P}_{12}, \mathbb{P}_{13}$ and $\mathbb{P}_{23}$ are defined in (18). Then

$$
\begin{aligned}
\chi_{3}(\lambda)= & 1-\frac{2 p_{1}}{\lambda-p_{1}} i a_{1} e^{\Delta F_{1}} \chi_{3}\left(q_{1}\right)-\frac{2 q_{1}}{q_{1}+\lambda} i a_{1} e^{\Delta F_{1}} \chi_{3}\left(-p_{1}\right)-\frac{2 p_{2}}{\lambda-p_{2}} i a_{2} e^{\Delta F_{2}} \chi_{3}\left(q_{2}\right) \\
& -\frac{2 q_{2}}{q_{2}+\lambda} i a_{2} e^{\Delta F_{2}} \chi_{3}\left(-p_{2}\right)-\frac{2 p_{3}}{\lambda-p_{3}} i a_{3} e^{\Delta F_{3}} \chi_{3}\left(q_{3}\right)-\frac{2 q_{3}}{q_{3}+\lambda} i a_{3} e^{\Delta F_{3}} \chi_{3}\left(-p_{3}\right) .
\end{aligned}
$$

\section{Concluding remarks}

In this paper, we have used the extended Moutard transformation to construct the $N$-solitons solutions. The basic idea comes from the successive iterations of solitons solutions, as remains to be the simple method to obtain the $N$-solitons solutions. Also, the corresponding wave functions are constructed by the Pfaffian expansion of the sum of two anti-symmetric matrices (14) when compared with the $\bar{\partial}$-dressing method [2].

To obtain real $N$-solitons solutions of the Novikov-Veselov equation (1), one has to put extra relations between $-p_{i}$ and $q_{i}$ [2]. It could be interesting to investigate these real solutions for the Schrödinger operator (self-adjoint). On the other hand, the resonance of $N$-solitons solutions of DKP or KP theory has been studied in $[16,17,18,19]$. And then the resonance of $N$-solitons solutions of Pfaffian type (15) deserves to be investigated. These issues will be published elsewhere.

\section{Acknowledgements}

This work is supported in part by the National Science Council of Taiwan under Grant No. 100-2115-M-606-001. 


\section{References}

[1] Athorne C., Nimmo J.J.C., On the Moutard transformation for integrable partial differential equations, Inverse Problems 7 (1991), 809-826.

[2] Basalaev M.Yu., Dubrovsky V.G., Topovsky A.V., New exact solutions with constant asymptotic values at infinity of the NVN integrable nonlinear evolution equation via $\bar{\partial}$-dressing method, arXiv:0912.2155.

[3] Bogdanov L.V., Veselov-Novikov equation as a natural two-dimensional generalization of the Korteweg-de Vries equation, Theoret. Math. Phys. 70 (1987), 219-223.

[4] Chang J.H., The Gould-Hopper polynomials in the Novikov-Veselov equation, J. Math. Phys. 52 (2011), 092703, 15 pages, arXiv:1011.1614.

[5] Dubrovin B.A., Krichever I.M., Novikov S.P., The Schrödinger equation in a periodic field and Riemann surfaces, Sov. Math. Dokl. 17 (1976), 947-952.

[6] Dubrovsky V.G., Formusatik I.B., New lumps of Veselov-Novikov integrable nonlinear equation and new exact rational potentials of two-dimensional stationary Schrödinger equation via $\bar{\partial}$-dressing method, Phys. Lett. A 313 (2003), 68-76.

[7] Dubrovsky V.G., Formusatik I.B., The construction of exact rational solutions with constant asymptotic values at infinity of two-dimensional NVN integrable nonlinear evolution equations via the $\bar{\partial}$-dressing method, J. Phys. A: Math.Gen. 34 (2001), 1837-1851.

[8] Grinevich P.G., Rational solitons of the Veselov-Novikov equations are reflectionless two-dimensional potentials at fixed energy, Theoret. Math. Phys. 69 (1986), 1170-1172.

[9] Grinevich P.G., Manakov S.V., Inverse scattering problem for the two-dimensional Schrödinger operator, the $\bar{\partial}$-method and nonlinear equations, Funct. Anal. Appl. 20 (1986), 94-103.

[10] Grinevich P.G., Mironov A.E., Novikov S.P., New reductions and nonlinear systems for 2D Schrödinger operators, arXiv:1001.4300.

[11] Hirota R., The direct method in soliton theory, Cambridge Tracts in Mathematics, Vol. 155, Cambridge University Press, Cambridge, 2004.

[12] Hu H.C., Lou S.Y., Construction of the Darboux transformaiton and solutions to the modified NizhnikNovikov-Veselov equation, Chinese Phys. Lett. 21 (2004), 2073-2076.

[13] Hu H.C., Lou S.Y., Liu Q.P., Darboux transformation and variable separation approach: the NizhnikNovikov-Veselov equation, Chinese Phys. Lett. 20 (2003), 1413-1415, nlin.SI/0210012.

[14] Ishikawa M., Wakayama M., Applications of minor-summation formula. II. Pfaffians and Schur polynomials, J. Combin. Theory Ser. A 88 (1999), 136-157.

[15] Kaptsov O.V., Shan'ko Yu.V., Trilinear representation and the Moutard transformation for the Tzitzéica equation, solv-int/9704014.

[16] Kodama Y., KP solitons in shallow water, J. Phys. A: Math. Gen. 43 (2010), 434004, 54 pages, arXiv:1004.4607.

[17] Kodama Y., Maruno K., N-soliton solutions to the DKP equation and Weyl group actions, J. Phys. A: Math. Gen. 39 (2006), 4063-4086, nlin.SI/0602031.

[18] Kodama Y., Williams L.K., KP solitons and total positivity for the Grassmannian, arXiv:1106.0023.

[19] Kodama Y., Williams L.K., KP solitons, total positivity, and cluster algebras, Proc. Natl. Acad. Sci. USA 108 (2011), 8984-8989, arXiv:1105.4170.

[20] Konopelchenko B.G., Introduction to multidimensional integrable equations. The inverse spectral transform in $2+1$ dimensions, Plenum Press, New York, 1992.

[21] Konopelchenko B.G., Landolfi G., Induced surfaces and their integrable dynamics. II. Generalized Weierstrass representations in 4D spaces and deformations via DS hierarchy, Stud. Appl. Math. 104 (2000), $129-169$.

[22] Krichever I.M., A characterization of Prym varieties, Int. Math. Res. Not. 2006 (2006), Art. ID 81476, 36 pages, math.AG/0506238.

[23] Liu S.Q., Wu C.Z., Zhang Y., On the Drinfeld-Sokolov hierarchies of D type, Int. Math. Res. Not. 2011 (2011), 1952-1996, arXiv:0912.5273.

[24] Manakov S.V., The method of the inverse scattering problem, and two-dimensional evolution equations, Russian Math. Surveys 31 (1976), no. 5, 245-246. 
[25] Matveev V.B., Salle M.A., Darboux transformations and solitons, Springer Series in Nonlinear Dynamics, Springer-Verlag, Berlin, 1991.

[26] Mironov A.E., A relationship between symmetries of the Tzitzéica equation and the Veselov-Novikov hierarchy, Math. Notes $\mathbf{8 2}$ (2007), 569-572.

[27] Mironov A.E., Finite-gap minimal Lagrangian surfaces in $\mathbb{C P}^{2}$, in Riemann Surfaces, Harmonic Maps and Visualization, OCAMI Stud., Vol. 3, Osaka Munic. Univ. Press, Osaka, 2010, 185-196, arXiv:1005.3402.

[28] Mironov A.E., The Veselov-Novikov hierarchy of equations, and integrable deformations of minimal Lagrangian tori in $\mathbb{C P}^{2}$, Sib. Electron. Math. Rep. 1 (2004), 38-46, math.DG/0607700.

[29] Moutard M., Note sur les équations différentielles linéaires du second ordre, C.R. Acad. Sci. Paris 80 (1875), $729-733$.

[30] Moutard M., Sur la construction des équations de la forme $\frac{1}{z} \frac{\partial^{2} z}{\partial x \partial y}=\lambda(x y)$, qui admettent une intégrale générale explicite, J. de. l'Éc. Polyt. 28 (1878), 1-12.

[31] Nimmo J.J.C., Darboux transformations in $(2+1)$-dimensions, in Applications of Analytic and Geometric Methods to Nonlinear Differential Equations (Exeter, 1992), NATO Adv. Sci. Inst. Ser. C Math. Phys. Sci., Vol. 413, Kluwer Acad. Publ., Dordrecht, 1993, 183-192.

[32] Nimmo J.J.C., Hall-Littlewood symmetric functions and the BKP equation, J. Phys. A: Math. Gen. 23 (1990), 751-760.

[33] Novikov S.P., Two-dimensional Schrödinger operators in periodic fields, J. Sov. Math. 28 (1985), 1-20.

[34] Novikov S.P., Veselov A.P., Two-dimensional Schrödinger operator: inverse scattering transform and evolutional equations, Phys. D 18 (1986), 267-273.

[35] Ohta Y., Pfaffian solutions for the Veselov-Novikov equation, J. Phys. Soc. Japan 61 (1992), 3928-3933.

[36] Orlov A.Yu., Shiota T., Takasaki K., Pfaffian structures and certain solutions to BKP hierarchies. I. Sums over partitions, arXiv:1201.4518.

[37] Shiota T., Prym varieties and soliton equations, in Infinite-Dimensional Lie Algebras and Groups (LuminyMarseille, 1988), Adv. Ser. Math. Phys., Vol. 7, World Sci. Publ., Teaneck, NJ, 1989, 407-448.

[38] Stembridge J.R., Nonintersecting paths, Pfaffians, and plane partitions, Adv. Math. 83 (1990), 96-131.

[39] Taimanov I.A., Tsarev S.P., Two-dimensional rational solitons and their blowup via the Moutard transformation, Theoret. Math. Phys. 157 (2008), 1525-1541, arXiv:0801.3225.

[40] Takasaki K., Dispersionless Hirota equations of two-component BKP hierarchy, SIGMA 2 (2006), 057, 22 pages, nlin.SI/0604003.

[41] Veselov A.P., Novikov S.P., Finite-zone, two-dimensional, potential Schrödinger operators. Explicit formulas and evolution equations, Sov. Math. Dokl. 30 (1984), 588-591. 